\title{
Transitive decompositions of graphs
}

\author{
GEOFFREY PEARCE
}

(Received 10 March 2008)

\begin{abstract}
A transitive decomposition of a graph is a partition of the arc set such that there exists a group of automorphisms of the graph which preserves and acts transitively on the partition. This turns out to be a very broad idea, with several striking connections with other areas of mathematics. In this thesis we first develop some general theory of transitive decompositions, and in particular we illustrate some of the more interesting connections with certain combinatorial and geometric structures. We then give complete, or nearly complete, structural characterizations of certain classes of transitive decompositions preserved by a group with a rank 3 action on vertices (such a group has exactly two orbits on ordered pairs of distinct vertices). The main classes of rank 3 groups we study (namely those which are imprimitive, or primitive of grid type) are derived in some way from 2-transitive groups (that is, groups which are transitive on ordered pairs of distinct vertices), and the results we achieve make use of the classification by Sibley [1] of transitive decompositions preserved by a 2-transitive group.
\end{abstract}

\section{References}

[1] T. Q. Sibley, 'On classifying finite edge colored graphs with two transitive automorphism groups', J. Combin. Theory Ser. B 90 (2004), 121-138.

GEOFFREY PEARCE, 79 Vincent St, Nedlands WA 6009, Australia e-mail: gpearce@student.uwa.edu.au

Thesis submitted to The University of Western Australia, May 2007. Degree approved, February 2008. Supervisors: Professor Cheryl E. Praeger and Dr John Bamberg.

(c) 2008 Australian Mathematical Society 0004-9727/08 \$A2.00+0.00 